\title{
Philosophiques
}

\section{Principes de pragmatique formelle du discours}

\section{Daniel Vanderveken}

Volume 34, numéro 2, automne 2007

URI : https://id.erudit.org/iderudit/015880ar

DOI : https://doi.org/10.7202/015880ar

Aller au sommaire du numéro

\section{Éditeur(s)}

Société de philosophie du Québec

\section{ISSN}

0316-2923 (imprimé)

1492-1391 (numérique)

Découvrir la revue

\section{Citer cet article}

Vanderveken, D. (2007). Principes de pragmatique formelle du discours. Philosophiques, 34(2), 229-258. https://doi.org/10.7202/015880ar

\section{Résumé de l'article}

Pourrait-on enrichir la théorie des actes de langage pour traiter du discours? Wittgenstein et Searle ont signalé des difficultés. Beaucoup de discours n'ont pas de but conversationnel, leur arrière-plan est indéfiniment ouvert, ils contiennent des énonciations dépourvues de pertinence et de félicité, et ainsi de suite. À mes yeux, l'objectif principal de la pragmatique du discours est d'analyser la structure et la dynamique des jeux de langage à but conversationnel. Pareils jeux de langage sont indispensables à tout genre de discours. La logique peut analyser leurs conditions de félicité car leur poursuite obéit à des règles constitutives. Beaucoup d'énonciations ne sont pas littérales ou sérieuses. Les unités de la conversation sont les actes illocutoires tentés, qu'ils soient littéraux, sérieux ou non. Comme Montague, je préconise l'usage de formalismes logiques en pragmatique. J'expliquerai comment il convient de réviser et développer les logiques intensionnelle et illocutoire, la logique des attitudes et de l'action afin de modéliser notre capacité de dialoguer. Je comparerai mon approche à d'autres (Austin, Belnap, Grice, Montague, Searle, Sperber et Wilson, Kamp, Wittgenstein) sur le plan de la méthodologie, des hypothèses et des enjeux. 


\title{
Principes de pragmatique formelle du discours
}

\author{
DANIEL VANDERVEKEN \\ (Université du Québec, Trois-Rivières) \\ www.vanderveken.org
}

\begin{abstract}
RÉSUMÉ. - Pourrait-on enrichir la théorie des actes de langage pour traiter du discours? Wittgenstein et Searle ont signalé des difficultés. Beaucoup de discours n'ont pas de but conversationnel, leur arrière-plan est indéfiniment ouvert, ils contiennent des énonciations dépourvues de pertinence et de félicité, et ainsi de suite. À mes yeux, l'objectif principal de la pragmatique du discours est d'analyser la structure et la dynamique des jeux de langage à but conversationnel. Pareils jeux de langage sont indispensables à tout genre de discours. La logique peut analyser leurs conditions de félicité car leur poursuite obéit à des règles constitutives. Beaucoup d'énonciations ne sont pas littérales ou sérieuses. Les unités de la conversation sont les actes illocutoires tentés, qu'ils soient littéraux, sérieux ou non. Comme Montague, je préconise l'usage de formalismes logiques en pragmatique. J'expliquerai comment il convient de réviser et développer les logiques intensionnelle et illocutoire, la logique des attitudes et de l'action afin de modéliser notre capacité de dialoguer. Je comparerai mon approche à d'autres (Austin, Belnap, Grice, Montague, Searle, Sperber et Wilson, Kamp, Wittgenstein) sur le plan de la méthodologie, des hypothèses et des enjeux.
\end{abstract}

\begin{abstract}
Could we enrich speech-act theory to deal with discourse? Wittgenstein and Searle pointed out difficulties. Most dialogues lack a conversational purpose, their background is indefinitely open, they contain irrelevant and infelicitous utterances, etc. In my view, the primary aim of discourse pragmatics is to analyze the structure and dynamics of language-games whose type is provided with an internal conversational goal. Such games are indispensable to any kind of discourse. Logic can analyze their felicity-conditions because they are conducted according to systems of constitutive rules. Speakers often speak non-literally or nonseriously. The units of conversation are attempted illocutions whether literal, serious or not. I will show how to construct speaker-meaning from sentencemeaning, conversational background and maxims. Like Montague, I believe that we need the resources of formalisms (proof-, model- and game-theories) and of logic in pragmatics. I will explain how to further develop intensional and illocutionary logics, the logic of attitudes and of action in order to characterize our ability to converse. I will compare my approach to others (Austin, Belnap, Grice, Montague, Searle, Sperber and Wilson, Kamp, Wittgenstein) as regards hypotheses, methodology and issues.
\end{abstract}

\section{Introduction}

Dans les sciences du langage, de la pensée et de l'action, on reconnaît à présent le rôle essentiel en toute communication des actes de discours, comme les actes d'énonciation, de référence et de prédication, les actes illocutoires et perlocutoires, ainsi que des attitudes des interlocuteurs comme leurs croyances, désirs et intentions. On a aussi compris l'importance cruciale des jeux de langage que les interlocuteurs entendent poursuivre ensemble, des formes de vie qu'ils 
partagent et de leur arrière-plan conversationnel. Selon la théorie des actes de discours, les unités premières de signification et de communication dans l'usage et la compréhension du langage ne sont pas des propositions isolées pourvues de conditions de vérité mais plutôt des actes illocutoires pourvus de conditions de félicité (Austin, 1962). Les actes illocutoires élémentaires ont une force (Frege, 1977) et un contenu propositionnel. Les interlocuteurs entendent toujours accomplir et communiquer des illocutions. Jusqu'à présent, la logique, la philosophie et la linguistique ont principalement étudié la capacité des interlocuteurs d'utiliser et de comprendre les seuls énoncés sans prendre beaucoup en considération leur capacité de tenir des discours entiers. Dans le même ordre d'idées, la théorie des actes de langage a surtout étudié les actes illocutoires individuels comme les assertions, les promesses et les requêtes que les interlocuteurs entendent accomplir en utilisant des énoncés à des moments d'énonciation particuliers. Pourtant, les interlocuteurs dialoguent le plus souvent avec d'autres agents. Avant tout, l'usage du langage est une forme sociale de comportement linguistique. Il en résulte des conversations orales et des textes écrits.

Peut-on étendre la théorie des actes de langage pour traiter de discours entiers? Wittgenstein et Searle ont exprimé leur scepticisme. Les interlocuteurs ont souvent des objectifs extralinguistiques, ils sont engagés dans des activités communes, et leur arrière-plan est indéfiniment ouvert. Ils font des énonciations sans félicité ni pertinence sans que cela interrompe pour autant leur dialogue. Ils changent aussi leurs objectifs de façon assez arbitraire. Ainsi l'exigence de pertinence impose relativement peu de contraintes à la structure de maintes conversations. Cependant les langues naturelles sont des langues publiques, et la capacité de dialoguer fait sans nul doute partie de la compétence linguistique. Qui plus est, les protagonistes accomplissent toujours en tout discours (que ce soit un dialogue ou un monologue) des actes illocutoires capitaux afin d'atteindre des buts proprement linguistiques correspondant à une direction possible d'ajustement entre les mots et les choses. Ils manifestent des attitudes communes (but expressif), ils décrivent comment les choses sont dans le monde (but descriptif), ils délibèrent comment y agir (but délibératif) et ils font des choses avec leurs mots par déclaration (but déclaratoire). De tels objectifs linguistiques sont purement conversationnels. Des interventions discursives comme les échanges de salutations, de bienvenues et de nouvelles, les débats, les entrevues, les consultations, les négociations, les compromis, les prises de position et de décision, les contrats ainsi que les cérémonies d'inauguration, de mariage et de baptême sont des actes illocutoires conjoints d'un niveau supérieur, irréductibles à des séquences d'illocutions individuelles instantanées. Plusieurs agents obéissant à des règles constitutives y contribuent chacun à leur tour durant un intervalle de temps. D'un point de vue logique, pareilles interventions possèdent un type et un thème, et leur poursuite requiert de la coopération. La logique du discours, comme je la conçois, n'entend pas étudier tous les jeux de langage possibles, mais seulement ceux dont l'objectif 
propre est linguistique. Il existe un grand nombre de pareils jeux de langage et ils sont indispensables à la poursuite de tout discours. Les interlocuteurs doivent tout d'abord se reconnaître en tant que protagonistes de la conversation et se mettre d'accord sur le genre de conversation en cours. Lorsqu'ils communiquent pour exécuter des tâches complexes, il leur faut tôt ou tard décrire leurs objectifs et délibérer comment procéder pour les atteindre. Le but de cet article est de présenter mon approche théorique en pragmatique du discours et de la comparer aux autres approches en cours quant à la méthodologie, aux hypothèses et à la problématique. Je tiendrai compte des travaux antérieurs en philosophie analytique (sur l'action, les attitudes et les actes de langage), en philosophie sociale (sur la coopération), en logique philosophique (sur le sens, la dénotation, les forces, les attitudes et les actions), en sémantique formelle (en idéographie logique, grammaire universelle et théorie de la représentation du discours), en analyse de la conversation (sur les maximes, l'arrière-plan et les genres conversationnels), en linguistique (sur les marqueurs illocutoires et les verbes d'action) et en psychologie (sur la compréhension mutuelle).

\section{Enjeux et objectifs théoriques}

À l'instar de Montague (1974), je pense que la pragmatique doit utiliser les ressources des formalismes et de la logique afin d'élaborer une théorie rigoureuse de la signification et de l'usage. Les langues naturelles peuvent être apprises par des agents humains dont les capacités cognitives sont créatives mais limitées (Chomsky). Les formalismes permettent de construire un meilleur modèle théorique de notre compétence linguistique et du mécanisme de la compréhension. Cependant, il convient de réviser de nombreuses hypothèses de la logique standard des propositions, des attitudes et de l'action afin de bien expliquer les conditions de félicité des illocutions. Les propositions avec les mêmes conditions de vérité ne sont pas les contenus des mêmes attitudes et illocutions, comme elles ne sont pas non plus les sens d'énoncés synonymes. On peut affirmer et croire que Paris est une ville sans pour autant affirmer et croire que c'est une ville et non un érythrocyte. La théorie des actes de langage requiert un critère plus fin d'identité propositionnelle. Elle requiert aussi une explication de l'intentionnalité et de la rationalité des interlocuteurs ainsi que de l'engendrement des différentes espèces d'actes de langage en logique de l'action. Il faut en outre intégrer la logique illocutoire dans l'idéographie de la sémantique formelle afin de clarifier la forme canonique des actes illocutoires exprimés par tous les types syntaxiques d'énoncés.

Bien entendu, les unités premières réelles de la conversation sont les actes illocutoires qui sont tentés plutôt que ceux qui sont exprimés. La signification du locuteur est souvent différente de celle de l'énoncé utilisé. En cas d'ironie, de métaphore ou d'indirection, les locuteurs ne parlent pas littéralement, tout comme ils ne parlent pas sérieusement quand ils jouent dans une pièce de théâtre. Afin de contribuer à la pragmatique du discours, la théorie 
des actes de langage devrait expliquer pleinement la signification du locuteur (tous les actes illocutoires tentés, qu'ils soient littéraux, sérieux ou non) ainsi que la structure et la dynamique des dialogues que les interlocuteurs sont aptes à poursuivre en vertu de leur compétence. Pour y parvenir, il faut une véritable théorie des maximes conversationnelles (Grice, 1975) et des faits pertinents à l'arrière-plan conversationnel. Les maximes de Grice sont malheureusement vagues et restreintes; elles ne s'appliquent qu'aux énonciations assertives dans les discours à but informatif. Il convient de généraliser l'approche de Grice et de mieux expliquer la pertinence que ne l'ont fait Sperber et Wilson (1986) qui négligent à la fois les forces illocutoires et les buts conversationnels. Il convient en outre d'analyser ce que font les interlocuteurs quand ils ne parlent pas sérieusement mais feignent d'accomplir des actes illocutoires, au théâtre comme dans la fiction. Afin de modéliser la dynamique de la poursuite du discours, on devrait enfin exploiter les ressources de la théorie des jeux (von Neumann) et profiter des développements récents en logiques dynamique, dialogique, paracohérente et non monotone ainsi qu'en théories de la représentation du discours et de la décision.

Depuis Montague, de nombreux logiciens et linguistes ont exploité des formalismes comme la théorie de la preuve et des modèles conçus originellement pour les langues artificielles afin d'élaborer une sémantique formelle rigoureuse des langues naturelles. Certains ont indirectement interprété les énoncés ordinaires après les avoir traduits dans une langue objet formelle désambiguïsée. Les idéographies permettent d'exhiber la structure logique profonde commune à toutes les langues naturelles et de définir récursivement la signification du locuteur et la compréhension de l'auditeur. Cependant, les unités premières de la signification et de la communication ne sont pas des propositions isolées, comme l'affirme Montague, mais plutôt des actes illocutoires élémentaires comme des assertions, des engagements, des questions, des nominations et des remerciements qui possèdent une force en plus d'un contenu propositionnel. Les locuteurs qui signifient quelque chose tentent d'accomplir des actes illocutoires. Voilà pourquoi il $\mathrm{y}$ a des marqueurs de force dans les énoncés élémentaires dont la forme logique est complètement analysée. Le type syntaxique des énoncés élémentaires est leur marqueur de force de base. À chaque fois qu'ils sont utilisés littéralement, les énoncés déclaratifs servent à faire des assertions, les énoncés interrogatifs à poser des questions, les énoncés impératifs à donner des directives et les énoncés optatifs à exprimer des souhaits. Ainsi, les énoncés avec des clauses synonymes comme "Tu viens m'aider », «Viens-tu m'aider ? », «S'il te plaît, viens m’aider ! » et « Si seulement tu venais m'aider » s'expriment dans les mêmes contextes des actes illocutoires avec le même contenu propositionnel mais avec des forces différentes. De même, des énoncés avec les mêmes marqueurs de force tels que «Viens! » et « Mange! » expriment des actes illocutoires avec la même force mais des contenus différents. Les grammairiens ont depuis fort longtemps reconnu les aspects illocutoires de la signification linguistique dans leur classification des types syntaxique 
d'énoncés. Grâce à la théorie des actes de langage, on peut dorénavant mieux analyser la signification des marqueurs et verbes illocutoires des langues naturelles. Les énoncés impératifs comme "Allons-y! », dont le verbe est à la première personne du pluriel, servent à offrir à l'interlocuteur de réaliser une action commune. Des énoncés explicitement performatifs comme « Par la présente je vous promets de l'aide » et « Je vous ordonne de sortir » servent à déclarer plutôt qu'à simplement affirmer que le locuteur effectue au moment de l'énonciation l'acte illocutoire nommé par leur verbe performatif. Comme les déclarations réussies rendent vraies le contenu propositionnel, les énonciations performatives réussies ont la force première de déclaration en plus de la force secondaire nommée par leur verbe performatif.

Jusqu'à présent, la logique traditionnelle a surtout étudié les conditions de vérité et les inférences théoriquement valides. Ainsi, la sémantique formelle a principalement analysé des expressions comme les noms propres, les descriptions, les prédicats, les connecteurs et les quantificateurs dont la signification contribue à la détermination des conditions de vérité et elle a tenté de construire la compétence linguistique comme étant la capacité des locuteurs de comprendre les contenus propositionnels. Selon Davidson et Montague, le seul objectif vraiment important de la sémantique est de formuler la théorie de la vérité. Pour cette raison, la plupart des applications de la sémantique formelle aux langues naturelles existantes ont été restreintes à l'interprétation de leurs énoncés déclaratifs. Cependant, le langage sert à changer le monde autant qu'à le décrire. Il y a cinq buts illocutoires que les locuteurs peuvent atteindre sur les contenus propositionnels qu'ils expriment (Searle, 1979). Le but assertif qui leur sert à représenter ce qui se passe dans le monde, le but engageant qui leur sert à s'engager eux-mêmes à y faire des actions, le but directif qui leur sert à essayer de faire agir les auditeurs, le but déclaratoire qui leur sert à changer des choses avec leurs mots, et le but expressif qui leur sert à exprimer les attitudes que leur inspirent des faits. Les locuteurs font en parlant des inférences pratiques autant que théoriques. Dans le cas des inférences théoriques ils tirent comme conclusions des actes illocutoires assertifs qui sont vrais ou faux. Quiconque affirme la conjonction de deux propositions affirme chacune d'entre elles. Les conclusions tirées d'inférences pratiques sont des actes illocutoires engageants, directifs ou déclaratoires représentant des actions du locuteur ou de l'auditeur. Quiconque pose une question tente d'obtenir une réponse de l'auditeur. Grâce à la logique illocutoire, la sémantique formelle devient capable d'analyser les marqueurs de force dont la signification contribue à la détermination des conditions de félicité et d'interpréter, sans la moindre réduction ad hoc aux énoncés déclaratifs, les autres types d'énoncés impératifs, interrogatifs, performatifs, exclamatoires, subjonctifs et autres, exprimant des actes illocutoires de force non assertive. Dans mon optique, la compétence linguistique est inséparable de la performance. Elle est avant tout la capacité des interlocuteurs d'accomplir et de comprendre les actes illocutoires. 
Par nature, les actes illocutoires sont des actions intrinsèquement intentionnelles: leur accomplissement requiert une tentative verbale du locuteur. Ils ont de véritables conditions de succès. Pour les réussir, les locuteurs doivent exprimer correctement les actes illocutoires tentés en utilisant les mots appropriés et en faisant leur tentative en un contexte adéquat. Un baptême n'est pas réussi quand le prêtre se trompe de nom propre ou de bébé. Les locuteurs lient les contenus propositionnels au monde avec l'intention d'établir une correspondance entre les mots et les choses selon une certaine direction d'ajustement. Quiconque fait une assertion entend représenter comment les choses sont dans le monde. Quiconque donne un ordre entend que l'auditeur fasse les choses représentées. Quiconque fait une déclaration entend faire les choses représentées avec ses propres mots. Les énonciations assertives ont la direction d'ajustement des mots aux choses, les énonciations engageantes et directives ont la direction d'ajustement des choses aux mots, et les déclarations ont la double direction d'ajustement. Pareils actes illocutoires ont en outre des conditions de satisfaction. Pour qu'un acte illocutoire élémentaire soit satisfait, il ne suffit pas que son contenu propositionnel soit vrai et corresponde à un fait existant dans le monde. La correspondance entre les mots et les choses doit être établie selon la bonne direction d'ajustement. Quiconque obéit à un ordre doit faire ce qui est ordonné à cause de cet ordre. S'il ne le fait pas pour cette raison, il n'obéit pas alors à l'ordre. La notion de satisfaction est une généralisation de celle de vérité qui couvre toutes les forces. Ainsi, une assertion est satisfaite quand elle est vraie; une promesse l'est quand elle est tenue; une requête quand elle est accordée et une déclaration quand elle rend vrai son contenu propositionnel. La satisfaction implique la causalité intentionnelle quand les forces ont la direction d'ajustement des choses aux mots.

Certains actes illocutoires réussis ne sont pas satisfaits. Des assertions peuvent être fausses, des promesses violées, des requêtes refusées et des ordres désobéis. Cependant, le succès, la satisfaction et la vérité sont logiquement reliés. La satisfaction d'actes illocutoires élémentaires requiert la vérité de leur contenu propositionnel. Certains actes illocutoires ont des conditions de succès ou de satisfaction plus fortes que d'autres. Quiconque supplie fait une requête. Quand une promesse est tenue, l'assertion de son contenu propositionnel est vraie. De plus, l'accomplissement de nombreux actes illocutoires exige la satisfaction d'autres. Les déclarations réussies contiennent des assertions vraies. Réciproquement, la satisfaction de certaines illocutions requiert l'accomplissement d'autres. On ne peut tenir qu'une promesse que l'on a faite. Quiconque comprend les conditions de félicité raisonne et fait des inférences pratiquement et théoriquement valides. Il comprend que certains actes illocutoires ne peuvent être accomplis et satisfaits sans que d'autres ne le soient également. Ainsi tout locuteur compétent infère d'une prémisse de la forme "Tout homme est mortel et Socrate est un homme» la conclusion assertive "Socrate est mortel " (inférence théorique). De la même façon, il infère d'une prémisse de la forme "Tu devrais aider autrui » la conclusion 
directive «Aide les autres!». Les conclusions tirées d'inférences théoriques ont la direction d'ajustement des mots aux choses, alors que les conclusions tirées d'inférences pratiques ont la direction d'ajustement des choses aux mots.

Il faut une théorie récursive unifiée du succès, de la satisfaction et la vérité pour expliquer tout cela. Dans ma perspective, la théorie sémantique de la vérité défendue par Montague et Davidson pour les langues naturelles fait partie de la théorie plus générale de la satisfaction pour les actes illocutoires de toute force. Les logiques antérieures des actes de discours, comme la logique des commandements de Rescher et la logique des questions de Belnap, et les tentatives d'étendre la logique intensionnelle à l'interprétation des énoncés non déclaratifs (Karttunen, Zaefferer, Stockoff) et d'énoncés dans un discours (Kamp) prétendent à tort que les conditions de succès et de satisfaction des actes illocutoires sont réductibles à leurs conditions de vérité. Elles ignorent les conditions réelles des conditions de félicité des actes de langage. La plupart des approches réduisent, par exemple, chaque question à un ensemble de réponses possibles à cette question et ensuite ces réponses à leur contenu référentiel ou propositionnel (Harrah).

\section{Progrès et méthodologie}

Dans Foundations of Illocutionary Logic (Searle et Vanderveken 1985), nous avons décomposé les forces en plusieurs composantes, à savoir : leurs buts illocutoires, leurs modes d'atteinte de but illocutoire, leurs conditions sur le contenu propositionnel, leurs conditions préparatoires et de sincérité et leur degré de puissance. Nous avons en outre défini récursivement l'ensemble des forces possibles ainsi que les conditions de succès des actes illocutoires de premier niveau que les locuteurs entendent accomplir à un moment d'énonciation donné. Les cinq forces primitives sont les forces les plus simples pourvues d'un but illocutoire propre: elles ont un degré de puissance neutre, et seulement les modes d'atteinte et les conditions qui sont déterminées par leur but. Ce sont: la force d'assertion nommée par le verbe performatif « affirmer » et exprimée par le type déclaratif d'énoncé; la force d'engagement à une action nommée par le performatif "s'engager à »; la force de toute tentative linguistique destinée à faire agir l'auditeur qui est exprimée par le type impératif d'énoncé; la force de déclaration nommée par le verbe performatif "déclarer » et exprimée lors d'énonciations performatives, et enfin la force d'expression d'attitudes réalisée dans le type exclamatif d'énoncé. Les cinq forces primitives sont universelles. On peut les exprimer en toute langue naturelle possible. Les autres forces illocutoires sont plus complexes; elles sont obtenues en ajoutant aux forces primitives de nouveaux modes spéciaux d'atteinte de but illocutoire, de nouvelles conditions sur le contenu propositionnel, de nouvelles préparatoires ou de sincérité, ou en changeant le degré de force.

D'un point de vue philosophique, les forces illocutoires actuelles de chaque langue sont des espèces naturelles d'emploi du langage. Elles remplissent des buts linguistiques importants pour les communautés humaines qui parlent 
cette langue dans leur environnement historique et naturel. Les composantes spéciales des forces complexes de chaque langue sont donc linguistiquement significatives pour sa communauté linguistique particulière à un certain moment de son histoire. Certaines sont transcendantes, car elles sont indispensables à toute langue humaine capable de remplir ses deux fonctions essentielles d'expression et de communication des pensées des agents. Telles sont les composantes des forces primitives. Telle est aussi la condition préparatoire que le fait représenté par le contenu propositionnel est bon ou mauvais. Car les locuteurs humains sont par nature des personnes avec des préoccupations éthiques. Les forces liées à des aspects historiques particuliers, par exemple la force déclaratoire d'excommunication qui dépend du sacrement de la communion, sont, elles, immanentes.

Les conditions de félicité des actes illocutoires élémentaires sont entièrement déterminées par leur force et leur contenu. Un locuteur accomplit avec succès un acte illocutoire de la forme $F(P)$ dont $F$ représente la force et $P$ le contenu propositionnel en un contexte donné d'énonciation quand, en ce contexte, premièrement, le locuteur réussit à atteindre le but illocutoire de cette force sur le contenu avec le mode d'atteinte qui lui est propre, deuxièmement, la proposition $P$ satisfait les conditions sur le contenu propositionnel de $F$, troisièmement, le locuteur présuppose alors toutes les propositions déterminées par les conditions préparatoires de cette force relativement au contenu et, finalement, il exprime également avec le degré de puissance requis par $F$ toutes les attitudes déterminées par les conditions de sincérité de cette force à propos de $P$. Ainsi un locuteur fait une promesse quand il se met alors lui-même dans l'obligation de faire une action (mode particulier d'atteinte du but illocutoire engageant et condition sur le contenu propositionnel), il présuppose que l'action est bonne pour l'auditeur (condition préparatoire) et il exprime une intention forte de la faire (degré de puissance et condition de sincérité). Un locuteur rate un acte illocutoire en un contexte quand il tente sans succès de l'accomplir. À la différence de l'insuccès. Le succès et l'échec contiennent une tentative de l'agent.

Il arrive que les locuteurs présupposent des propositions qui sont fausses et qu'ils expriment des attitudes qu'ils ne possèdent pas. Certaines promesses ne sont pas dans l'intérêt de l'auditeur. Les locuteurs font aussi des promesses qu'ils n'ont pas l'intention de tenir. Pareilles promesses sont défectueuses. Un acte illocutoire est sans défaut quand il est accompli avec succès et que toutes ses conditions préparatoires et de sincérité sont remplies. Austin, avec sa notion de condition de félicité n'a pas clairement distingué les énonciations réussies qui sont défectueuses des énonciations ratées qui ne sont même pas réussies. En logique illocutoire, un acte illocutoire est plein de félicité quand il est à la fois réussi, sans défaut et satisfait. John Searle et moi-même (1985) avons analysé la signification des marqueurs de force et des verbes performatifs de la langue anglaise et avons souligné la nature déclaratoire des énonciations performatives. De nombreux marqueurs de force contiennent des traits syntaxiques qui 
s'ajoutent au type syntaxique de l'énoncé et servent à exprimer une force particulière avec des composantes nouvelles. Des énoncés de même type syntaxique peuvent donc exprimer des actes illocutoires de force différente. Les énoncés impératifs comme «Faites-le s'il vous plaît! » et "Faites-le, que ça vous plaise ou non! » expriment respectivement une demande et un ordre. Les énoncés exclamatifs «Comme je suis content de cela! » et «Comme je suis triste de cela! » servent à exprimer des attitudes de modes psychologiques différents, respectivement du contentement et de la tristesse. Searle et moi n'avons pas analysé en détail les conditions de satisfaction des actes illocutoires.

À cette fin, j'ai formulé par la suite une logique prédicative naturelle des propositions tenant compte de leur double nature : les propositions sont à la fois des sens d'énoncés et des contenus d'actions et d'attitudes. Elles ont une structure de constituants : en exprimant des propositions, nous nous référons aux objets via des concepts et leur attribuons des propriétés et des relations. Nous n'avons pas directement à l'esprit les objets individuels ${ }^{1}$ comme les corps matériels et les personnes. Nous avons plutôt à l'esprit des concepts de ces individus et nous nous y référons indirectement par l'entremise de ces concepts. Nos pensées et nos actions sont donc dirigées vers des individus subsumés sous des concepts plutôt que vers de purs individus. Nous ignorons les valeurs de vérité de nombreuses propositions parce que nous ignorons les dénotations réelles de leurs constituants en de nombreuses circonstances. On peut se référer à l'épouse du maire sans savoir qui elle est. Pourtant, nous pouvons alors en principe penser à des personnes qui pourraient être cette épouse. Ainsi, dans tous les usages et interprétations du langage, il existe de nombreuses assignations possibles de dénotation aux attributs et aux concepts en plus de l'assignation standard réelle de dénotation qui associe aux constituants propositionnels leur véritable dénotation en chaque circonstance possible. Les assignations possibles de dénotation que je préconise en sémantique respectent les postulats de signification. Seuls des ensembles d'individus subsumés sous des concepts sont des dénotations possibles de propriétés de premier ordre en chaque circonstance. Les épouses sont des femmes mariées selon toutes les assignations possibles de dénotation. Selon l'assignation réelle, l'épouse du maire est la femme avec laquelle il est réellement marié quand il est marié. Selon d'autres assignations possibles, ce sont d'autres femmes ou personnes. Lors de la compréhension de propositions, nous savons simplement que leur vérité en chaque circonstance possible est compatible avec certaines assignations possibles de dénotation à leurs constituants et incompatibles avec les autres. Pour être vraie, une proposition doit, bien entendu, être vraie selon l'assignation réelle de dénotation. La logique prédicative explique pourquoi des propositions vraies dans les mêmes circonstances ont une valeur cognitive différente. Certaines ont différentes structures de constituants. Telles sont les propositions que les mères sont

1. Les objets individuels sont les entités de référence du type logique le plus simple. Voir Strawson (1959). 
des femmes et que les érythrocytes sont rouges. Leur expression requiert différents actes de prédication. Les autres ne sont pas vraies selon les mêmes assignations possibles de dénotation. Telles sont les propositions que les baleines sont des baleines et que les baleines sont des mammifères. Nous ne les comprenons pas comme étant vraies dans les mêmes conditions. Ainsi nous pouvons affirmer ou croire des vérités nécessaires sans en affirmer ou en croire d'autres. Parmi toutes les vérités nécessaires, peu sont de pures tautologies comme la proposition selon laquelle les baleines sont des baleines, qui sont vraies en toute circonstance possible selon toute assignation possible de dénotation. D'un point de vue épistémique, certaines assignations possibles de dénotation sont compatibles avec les croyances d'agents, d'autres ne le sont pas. Si par exemple un agent croit que l'épouse du maire est d'origine étrangère, toutes les assignations possibles de dénotation selon lesquelles elle ne l'est pas sont incompatibles avec ce qu'il croit alors.

Il n'existe pas de correspondance biunivoque entre les verbes performatifs ou les marqueurs de force d'une langue, d'une part, et les forces illocutoires possibles d'autre part. De nombreux verbes performatifs et certains marqueurs de force sont ambigus. On peut aussi bien jurer que quelque chose s'est passé (énonciation assertive) que jurer de faire quelque chose (énonciation engageante). Qui plus est, la structure de surface des marqueurs et des verbes illocutoires n'exhibe pas la forme logique des forces exprimées. Certains types syntaxiques d'énoncés comme les types déclaratif, impératif et exclamatif expriment les forces primitives assertive, directive et expressive, alors que d'autres comme les types conditionnel et interrogatif expriment des forces plus complexes avec des composantes additionnelles. Par exemple, une question est une directive avec une condition spéciale sur le contenu propositionnel, à savoir que l'auditeur donne une réponse correcte à la question. Tout comme on obtient les forces complexes en ajoutant à des forces plus simples des nouvelles composantes ou en changeant leur degré de puissance, on compose en chaque langue des marqueurs de forces complexes en ajoutant au type de l'énoncé des traits syntaxiques nouveaux. Les énoncés impératifs dont le verbe est modifié par l'adverbe "s'il vous plaît » expriment des demandes. Leur adverbe exprime le mode poli d'atteinte du but illocutoire directif : le locuteur donne alors l'option de refus à l'auditeur. Des énoncés comme «Heureusement il pleut » et «Hélas il pleut » expriment des forces assertives complexes ayant respectivement la condition préparatoire et la condition de sincérité exprimée par leur adverbe. Comme Montague l'a souligné, en traduisant les clauses des énoncés ordinaires dans la langue objet de la logique intensionnelle, la sémantique formelle clarifie la forme logique des propositions et parvient à une meilleure explication de leurs conditions de vérité. De même en traduisant les marqueurs de force et les verbes performatifs dans l'idéographie de la logique illocutoire, la sémantique formelle clarifie la forme logique des actes illocutoires et parvient à une meilleure explication de leurs conditions de félicité. 
Dans Meaning \& Speech Acts (Vanderveken, 1990-1991) j'ai élaboré une sémantique formelle générale du succès, de la satisfaction et de la vérité capable d'interpréter, après leur traduction dans l'idéographie, tous les types d'énoncés (déclaratifs ou non) et de formaliser la nature des inférences pratiques aussi bien que théoriques. Selon Cocchiarella dans le volume 10 de Routledge History of Philosophy, « la notion de lingua philosophica en tant que langue idéale contenant à la fois une logique intensionnelle et illocutoire n'est plus simplement un programme mais a d'ores et déjà été réalisée sous de nombreux aspects" (1997: 72). J'ai enrichi l'ontologie formelle de la sémantique en ajoutant les valeurs de succès (le succès et l'insuccès) aux valeurs de vérité (le vrai et le faux) et en adoptant une analyse prédicative des propositions. Les clauses avec des expressions indexicales expriment différentes propositions en différents contextes. Comme David Kaplan (1989), j'ai procédé à une double indexation sémantique selon laquelle la force et le sens aussi bien que la dénotation peuvent varier d'un contexte d'énonciation à un autre. J'ai ainsi distingué la signification linguistique de la signification en contexte. La signification linguistique d'un énoncé est, dans mon optique, une fonction associant des actes illocutoires aux énoncés en chaque contexte possible. La signification en un contexte d'un énoncé est l'acte illocutoire qu'il y exprime (peu importe que le locuteur l'utilise ou non en ce contexte). De nombreux énoncés expriment différents actes illocutoires en différents contextes, tout comme de nombreux actes illocutoires exprimés en un contexte ont différentes valeurs de succès et de satisfaction en des circonstances différentes. En outre, des énoncés qui ne sont pas synonymes peuvent exprimer le même acte illocutoire en différents contextes. Toute énonciation aujourd'hui de l'énoncé «Il pleut aujourd'hui à Montréal » exprime la même assertion que les énonciations hier de l'énoncé « Il pleuvra demain à Montréal».

Ainsi, il faut ramifier les notions sémantiques fondamentales d'analyticité, de cohérence et d'implication logique (entailement). Toute énonciation littérale de l'énoncé paradoxal de Moore (Wittgenstein, 1958, II, x), «Il va pleuvoir et je ne le crois pas ", est analytiquement ratée: cet énoncé ne peut être utilisé littéralement avec succès. Car aucun locuteur ne peut sans se contredire accomplir simultanément un acte illocutoire et dénier ses conditions de sincérité. Mais comme nous pouvons mentir, les énonciations littérales de l'énoncé de Moore ne sont pas analytiquement insatisfaites. Cet énoncé exprime une assertion vraie en chaque contexte où le locuteur affirme, sans être sincère, qu'il va pleuvoir. De plus, l'énoncé paradoxal de Moore est illocutoirement cohérent : il exprime une assertion performable. Quelqu'un d'autre peut affirmer qu'il va pleuvoir et que je ne le crois pas. Pour ce faire, il doit évidemment utiliser un autre énoncé que celui de Moore.

Comme il y a quatre relations différentes d'inclusion en logique illocutoire entre les conditions de succès et de satisfaction des actes illocutoires, il y a en sémantique générale quatre genres différents d'implication logique (d'entailement) entre les énoncés. Certains énoncés impliquent illocutoirement 
(ou vériconditionnellement) d'autres énoncés: ils expriment en chaque contexte un acte illocutoire dont le succès (ou la satisfaction) entraîne le succès (ou la satisfaction) des actes illocutoires exprimés par les autres énoncés dans le même contexte. L'énoncé performatif « Je demande votre aide » implique illocutoirement l'énoncé impératif « Aidez-moi s'il vous plaît! » et véri conditionnellement l'énoncé conditionnel "Vous pourriez m'aider ». Les notions de cohérence, d'analyticité et d'implication illocutoire ont été, par le passé, totalement ignorées en sémantique formelle. Pourtant, elles s'appliquent à tous les types d'énoncés et elles diffèrent des notions de cohérence, d'analyticité et d'implication vériconditionnelle. Les différentes notions ramifiées de la sémantique générale ne coïncident pas extensionnellement (voir Vanderveken, 2004). Ainsi les énoncés performatifs comme «Je vous ordonne de le faire » impliquent illocutoirement mais pas vériconditionnellement les énoncés non performatifs comme «Faites-le, que cela vous plaise ou non! » qui leur correspondent. Comme les déclarations ont la double direction d'ajustement, quiconque déclare avec succès qu'il accomplit un acte illocutoire au moment de l'énonciation accomplit alors ipso facto cet acte. Cependant, cet acte illocutoire secondaire n'est pas pour autant satisfait. L'interlocuteur peut désobéir à l'ordre donné.

En assignant comme valeurs sémantiques aux énoncés de chaque contexte des actes illocutoires complets avec leurs conditions de félicité, la sémantique analyse mieux la signification des différents types d'énoncés et les inférences valides qu'on peut faire en les utilisant. Elle établit les liens qui existent entre les énonciations de buts illocutoires différents. D’une part, les énonciations performatives sont du genre le plus fort, car les déclarations réussies sont également satisfaites et sans défaut. Leur succès implique leur félicité. Ce n'est pas sans raison qu'Austin a découvert les forces illocutoires grâce aux énoncés performatifs. Tous les actes illocutoires peuvent être accomplis par déclaration. Par conséquent, les énoncés performatifs impliquent illocutoirement tous les autres types d'énoncés, mais la réciproque n'est pas vraie. On peut accomplir un acte illocutoire sans le faire par déclaration. D'autre part, les énonciations purement expressives sont du genre le plus faible, car tous les actes illocutoires ont des conditions de sincérité. Les énoncés de chaque type impliquent donc illocutoirement les énoncés exclamatifs correspondants. Par exemple, l'énoncé exclamatif "Comme je suis triste qu'il soit mort! » est impliqué illocutoirement par le déclaratif « Hélas il est mort».

Nous devons enrichir la logique intensionnelle en traitant de l'action, du temps, des modalités et des attitudes afin de bien formuler notre théorie de la félicité. Les actes illocutoires des locuteurs sont des actions intrinsèquement intentionnelles reliées à d'autres actions verbales et non verbales ainsi qu'aux attitudes. C'est en utilisant des signes que les locuteurs tentent d'accomplir leurs actes illocutoires. Certains actes illocutoires ont même des contenus propositionnels qui représentent les actions présentes ou futures des interlocuteurs. Par exemple, les contenus propositionnels des engagements et des déclarations représentent des actions de leur locuteur et celles des directives des actions de 
leurs auditeurs. Notre idéographie plus riche donne à la sémantique formelle les moyens de mieux montrer après traduction les formes canoniques des actes illocutoires exprimés par tous les types d'énoncés (déclaratifs ou non) des langues naturelles. Indiscutablement, la force et le contenu des énonciations dépendent souvent des énonciations antérieures. Quiconque réplique à l'énonciation interrogative "Quel âge avez-vous ? » en spécifiant son âge fait plus qu'une assertion; il donne une réponse à la question posée. Nous avons besoin d'une représentation du discours (Kamp \& Reyle, 1993) afin de résoudre dynamiquement les ambiguiités, les ellipses, les anaphores, les présuppositions et les composantes de force dans le contexte de l'énonciation. Ainsi, la sémantique doit considérer, en plus du locuteur, du ou des auditeur(s), du moment et du lieu de chaque contexte possible d'énonciation, son arrière-plan conversationnel (les énonciations antérieures, les formes de vie et les attitudes partagées par les interlocuteurs, notamment leurs connaissances mutuelles).

Nous avons besoin d'une conception ramifiée du temps (Prior, 1967) et des modalités historiques (Thomason, 1984) afin rendre compte de l'indéterminisme du monde actuel et de la liberté des agents humains. Selon l'indéterminisme, la façon dont les choses sont à un moment n'est pas entièrement déterminée par la façon dont elles ont été auparavant. En particulier, nos actes de discours et nos autres actions ne sont pas déterminés. Lorsque nous disons et faisons quelque chose, nous aurions pu dire ou faire autre chose, ou ne rien dire ou faire du tout. Selon la conception ramifiée du temps, chaque moment représente un état complet possible du monde actuel à un instant donné. Plusieurs moments incompatibles peuvent succéder à un moment dans le futur de ce monde. Ce moment appartient alors à plusieurs histoires ayant le même passé et le même présent, mais différentes continuations historiques. Chaque histoire représente un des cours possibles de l'histoire du monde actuel. Comme Belnap (2001), je pense que les circonstances possibles où les propositions ont une valeur de vérité sont des paires d'un moment du temps et d'une histoire à laquelle ce moment appartient. Les propositions futures peuvent être vraies à un certain moment selon certaines continuations possibles de ce moment et fausses selon d'autres. Certaines histoires ont un moment final. Elles représentent un cours possible du monde qui se termine à ce moment final. Cependant, les moments qui ne sont pas finaux continuent toujours dans une seule direction. Comme Occam l'a souligné, parmi toutes les histoires auxquelles un moment appartient, il y en a une et une seule qui représente la marche du monde après ce moment (voir Prior, 1967). Aucun agent (même Dieu) ne sait à un moment donné quelle histoire sera sa continuation historique réelle, mais chacun est capable de distinguer cette histoire propre des autres et d'y faire référence pour représenter la valeur de satisfaction encore indéterminée des actes et illocutoires, et des attitudes propres aux locuteurs à ce moment. Dans mon optique (Vanderveken, 2006), les actes illocutoires et les attitudes ont une valeur de satisfaction propre dans chaque contexte, même si cette valeur est encore indéterminée quand leur contenu propositionnel est futur. Une prédiction 
faite maintenant sera vraie seulement si les choses seront telles que représentées dans la continuation historique réelle de ce monde, quelle que soit cette continuation. De même, une promesse faite au moment présent sera tenue seulement si son agent accomplit la chose promise dans la continuation réelle du monde. Les autres continuations historiques possibles n'importent pas.

Durant la dernière décennie, j'ai enrichi la logique propositionnelle prédicative en traitant de la généralisation, des modalités logiques et historiques, du temps ramifié et des attitudes aussi bien volitives que cognitives. J'ai aussi élaboré la logique de l'action intentionnelle. À cette fin, j'ai analysé les tentatives des agents qui sont des actions d'un genre spécifique: elles sont à la fois personnelles, intentionnelles, libres et réussies. Les agents font personnellement et volontairement leurs propres tentatives. Quiconque tente de faire une tentative la fait. Il aurait pu ne pas la faire. Les agents font leurs tentatives afin d'atteindre un autre objectif. Pour atteindre cet objectif, ils doivent faire une bonne tentative en une bonne circonstance. Quand un agent rate son objectif, sa tentative n'est pas satisfaite. Dans ma logique de l'action (2005), les actions intentionnelles sont premières, comme en philosophie. Les actions inintentionnelles des agents sont en effet engendrées par leurs actions intentionnelles et elles auraient aussi pu être tentées. Ainsi, les erreurs et les échecs ne sont pas des actions mais plutôt des événements qui nous arrivent. De plus, aucune action n'est inévitable. L'agent aurait pu ne pas la faire.

Ma théorie formule les lois gouvernant les tentatives des agents et elle explique la nature de leurs actions intentionnelles et de leurs actions de base. Elle rend aussi compte de la rationalité minimale des agents qui ne sont ni parfaitement rationnels ni entièrement irrationnels. Comme nous le verrons, notre rationalité minimale est reliée à la façon dont nous représentons les conditions de félicité. Il nous arrive de croire et de tenter de faire des choses impossibles. Nos possibilités subjectives ne sont donc pas toujours objectives. Cependant, nous ne pouvons ni croire ni tenter de faire des choses impossibles qui soient purement contradictoires. Mon explication des possibilités subjectives est basée sur la définition suivante de la vérité selon un agent: une proposition est vraie selon un agent dans une circonstance quand celui-ci a alors à l'esprit tous ses concepts et attributs et que cette proposition est vraie dans cette circonstance selon toutes les assignations possibles de dénotation qui sont alors compatibles avec ses croyances. Nous avons à l'esprit des propriétés sans savoir quelles entités les possèdent, tout en sachant bien que seules certaines entités pourraient les posséder. C'est pourquoi certaines, mais pas toutes, les assignations possibles de dénotation sont compatibles avec nos croyances. Nous apprenons a posteriori la plupart des propriétés essentielles des objets; j'entends par là des propriétés qu'ils possèdent réellement en toute circonstance possible comme par exemple que les baleines sont des mammifères. Nous ignorons bien des propriétés essentielles. Rares sont les agents qui connaissent leur code génétique. Des assignations possibles de dénotation compatibles avec nos croyances violent parfois des propriétés essentielles des objets auxquels nous pensons. 
Ainsi, il nous arrive d'être incohérents et de croire des propositions nécessairement fausses. Dans le temps, nous croyions que les baleines étaient des poissons. Cependant, toutes les assignations possibles de dénotation à l'œuvre dans l'usage et l'interprétation du langage respectent les postulats de signification. Voilà pourquoi nous sommes et restons à tout le moins minimalement rationnels. Quand une proposition est par définition vraie en toute circonstance possible selon toutes les assignations possibles de dénotation, nous savons a priori qu'elle est nécessairement vraie. Ainsi, nous ne pouvons exprimer de pures tautologies sans savoir leur vérité. Nous faisons les inférences théoriquement valides dont les prémisses impliquent fortement la conclusion. Une proposition en implique fortement une autre quand, premièrement, elle contient toutes ses prédications et, deuxièmement, elle l'implique tautologiquement en ce sens qu'il est tautologique que si elle est vraie, l'autre l'est également. Ma relation d'implication forte exige beaucoup plus que l'implication stricte de Lewis : elle est finie, paracohérente, tautologique, analytique et décidable. Elle est à la base des engagements psychologiques et illocutoires des locuteurs basés sur la compréhension des conditions de vérité. Cela permet d'améliorer la théorie de la déduction naturelle. Seules certaines règles de la déduction naturelle comme l'élimination de la disjonction engendrent de l'implication forte. Ainsi, une intention et une promesse de boire de la bière ou du vin contiennent respectivement une intention et une promesse de boire. Par contre, l'introduction de la disjonction n'engendre pas d'implication forte, puisque sa conclusion peut avoir de nouveaux sens. Une intention de boire ne contient pas une intention de boire ou de mourir.

Pour qu'agent réussisse à faire des choses, il ne suffit pas qu'il tente de les faire et qu'elles arrivent. Il faut que ces choses soient causées par sa propre tentative. Comment expliquer pleinement la causalité intentionnelle ? Souvent la tentative de l'agent est la cause même de l'état de choses tenté. Cependant parfois, il y a une surdétermination causale: les agents font certaines choses pour différentes raisons. La tentative des agents est alors simplement être une raison pratique de leur survenance. Il convient d'analyser la notion de raison pratique afin d'expliquer la causalité intentionnelle et les conditions de satisfaction des actes illocutoires dont la direction d'ajustement va des choses aux mots. Un auditeur obéit à un ordre quand cet ordre est une raison pratique pour laquelle il fait les choses ordonnées.

Grâce à la logique de l'action, du temps, des attitudes et des modalités, la logique illocutoire peut mieux analyser les contenus propositionnels et les forces. Son idéographie peut exprimer les actions passées, présentes et futures, les capacités et les attitudes des interlocuteurs. Elle peut aussi caractériser la façon dont les actes illocutoires sont reliés aux autres actes de langage (les actes d'énonciation, les actes propositionnels, les tentatives d'accomplir des actes illocutoires et les actes perlocutoires). Les tentatives visant à accomplir des actes illocutoires sont un nouvel acte de langage fort important dans ma taxonomie. Car elles sont constitutives de la signification. À mes yeux, une énonciation est 
pourvue de signification lorsque le locuteur tente d'accomplir un acte illocutoire, peu importe qu'il réussisse ou qu'il échoue. Les locuteurs tentent d'accomplir leurs actes illocutoires publics en émettant des signes. Il convient d'expliquer comment et en quelles conditions ils réussissent et de quelles façons leurs actes illocutoires réussis en engendrent d'autres (chaque invitation contient une requête) et ont des effets perlocutoires (l'auditeur est parfois influencé). À la base de toute communication, les agents tentent de mouvoir des membres de leur propre corps et cela engendre (Goldman, 1970) de diverses façons leurs actes de langage. Cet engendrement dans la communication est d'abord physiquement causal (nous utilisons oralement les énoncés en proférant des sons); il est ensuite conventionnel (la signification des énoncés sert à déterminer la nature des actes illocutoires exprimés). L'engendrement est parfois simple (les locuteurs mentent lorsqu'ils n'ont pas les attitudes exprimées) ou par extension (ils accomplissent parfois indirectement des actes illocutoires non littéraux). Afin d'expliquer l'engendrement des différents genres d'actes de langage, j'intégrerai la logique illocutoire dans le cadre de la logique de l'action.

Ma méthodologie est liée à mon effort de formalisation. Les notions théoriques primitives que j'ai choisies obéissent à des postulats de signification clairs et distincts et se prêtent à des axiomes évidents. Les autres notions importantes sont définissables à partir des primitives par élimination, par explication formelle ou par induction récursive. Les lois de l'idéographie devraient être valides selon la théorie des modèles et, autant que possible, prouvables voire décidables quand les agents les ont intériorisées par l'entremise de leur compétence. Je vise évidemment des axiomatisations aussi complètes que possibles et une théorie permettant un traitement linguistique et computationnel adéquats des langues naturelles.

\section{La signification des énoncés et la signification des locuteurs dans la conversation}

Comme Frege, je pense que la force, le sens et la dénotation sont les trois composantes de base de la signification des énoncés et des locuteurs. Quels actes illocutoires le locuteur entend-il accomplir dans le contexte d'une énonciation? La théorie des actes de langage adopte un principe de littéralité : elle identifie la signification du locuteur avec la signification de l'énoncé pour autant que cela soit possible. Dans cette optique, le locuteur tente, en premier lieu, d'accomplir l'acte illocutoire exprimé par l'énoncé dont il fait usage quand pareil acte est alors performable. Les langues naturelles offrent de riches moyens linguistiques pour exprimer les forces. De nombreux analystes négligeant ces moyens en viennent à ignorer les forces dans leur sémantique ou à multiplier inutilement les forces littérales. En suivant le principe d'économie d'Occam, Searle et moi, comme Grice, ne multiplions pas sans raison la signification des énoncés ni l'ambiguité sémantique. Nous faisons autant que possible appel aux maximes conversationnelles et à l'arrière-plan pour expliquer la signification non littérale du locuteur. Ainsi, les énoncés déclaratifs, interrogatifs et 
optatifs tels que " Je désire votre aide ", "Si seulement vous vouliez m'aider » et «Pouvez-vous m'aider ? " si souvent utilisés pour demander indirectement de l'aide expriment littéralement dans tout contexte, selon ma sémantique générale, respectivement une assertion, une question et un souhait. Comme Grice (1975) l'a souligné, la plupart des actes illocutoires non littéraux sont annulables. Dans d'autres contextes, le locuteur utilisant le même énoncé ne les accomplirait pas. Il en va ainsi pour les demandes récurrentes indirectes que l'on fait avec les énoncés précédents, lesquelles sont des implicatures conversationnelles généralisées dans la terminologie de Grice.

La plupart des énoncés peuvent être utilisés littéralement. Grâce à sa nouvelle logique propositionnelle et de l'action, ma sémantique générale dispose désormais d'une idéographie plus riche avec des capacités expressives plus grandes. Elle peut dorénavant mieux traduire les énoncés des langues naturelles et assigner aux actes illocutoires exprimés une forme canonique plus précise clarifiant leurs conditions de félicité. Dans mon optique, lors d'énonciations non littérales, les locuteurs expriment les actes illocutoires littéraux mais ils entendent en accomplir d'autres. Autrement, il y aurait tellement de sens et de forces qu'une véritable théorie de la signification linguistique serait impossible. Les locuteurs utilisant des énoncés illocutoires incohérents comme "Je ne suis pas moi-même aujourd'hui » savent, en vertu de leur compétence, que l'acte illocutoire littéral est imperformable. Ils ne signifient pas ce qu'ils disent. La sémantique générale qui traite du seul usage littéral n'est pas en mesure d'analyser les énonciations non littérales. Mais elle peut être intégrée dans une pragmatique systématique et unifiée plus générale capable de construire effectivement la signification non littérale des locuteurs à partir de la signification des énoncés utilisés et de l'arrière-plan conversationnel.

En vertu de sa forme logique, chaque acte illocutoire tenté engage le locuteur à de nombreux autres. Un acte illocutoire engage fortement le locuteur $\grave{a}$ un autre quand il ne peut alors accomplir cet acte sans ipso facto accomplir l'autre. Toutes les prédictions contiennent des assertions, mais la réciproque n'est pas vraie. Les assertions sur le passé ne sont pas des prédictions. Quiconque entend accomplir un acte illocutoire entend ipso facto accomplir les autres actes illocutoires ayant moins de conditions de succès. Mais les locuteurs sont aussi parfois engagés à des actes illocutoires plus forts. Celui qui réplique à une assertion en disant le contraire fait plus qu'affirmer la négation de son contenu. Il contredit alors le locuteur précédent. Ma sémantique formelle générale peut dorénavant expliquer par l'engendrement simple depareils engagements à des actes illocutoires plus forts. Dans les contextes où sont remplies des conditions sur le contenu propositionnel, des conditions préparatoires ou de sincérité spéciales, l'acte illocutoire littéral réussi engendre alors des actes illocutoires avec une force supérieure. Dans les autres contextes, il n’y a pas de tel engendrement illocutoire simple.

Comme Searle et moi l'avons signalé, les locuteurs sont aussi faiblement engagés à des actes illocutoires qu'ils n'accomplissent pas ouvertement. Celui 
qui accepte d'aider tout le monde est engagé à accepter de vous aider même s'il n'a fait aucune référence à vous. Nul ne peut littéralement dire « Je refuse de vous aider et j'accepte d'aider n'importe qui. » Les tentatives peuvent échouer. Mais quiconque tente d'accomplir un acte illocutoire est faiblement engagé à cet acte. Comment expliquer à quels actes de langage le locuteur est faiblement engagé ? Il existe une relation réflexive et symétrique de compatibilité agentive en logique de l'action. Deux moments sont compatibles eu égard à un agent quand toutes les actions qu'il a accomplies à ces deux moments sont simultanément performables. Cette relation permet de définir rigoureusement l'engagement illocutoire en théorie du succès. Un locuteur est faiblement engagé à un acte illocutoire, dans un certain contexte, quand il peut accomplir cet acte illocutoire à tout moment qui est compatible, en ce qui le concerne, avec le moment de l'énonciation de ce contexte. Toutes les lois gouvernant les engagements illocutoires faibles découlent de cette définition. Voilà une simplification majeure de Foundations où la notion d'engagement faible était primitive.

La signification du locuteur est souvent différente de la signification de l'énoncé dans le dialogue. Non seulement les interlocuteurs parlent souvent de façon non littérale ou non sérieuse, mais ils partagent des formes de vie et entendent en outre, par leurs interactions verbales, atteindre des objectifs communs et accomplir ensemble des actions. Comme Wittgenstein l'a souligné (1958), les énoncés sont des instruments qui possèdent des rôles et des fonctions à l'intérieur de jeux de langage. "Ici, le terme de ' jeu de langage' est un moyen de souligner le fait que l'usage du langage fait partie d'une activité, d'une forme de vie » (Wittgenstein, 1958: 23). Notre question est à présent: peut-on généraliser la théorie des actes de langage de façon à traiter de la conversation?

Avant tout, la poursuite d'une conversation est gouvernée par un principe général de coopération (Grice, 1975). Les interlocuteurs qui entendent contribuer à une conversation respectent certaines maximes conversationnelles comme «Dites le Vrai! », "Croyez ce que vous dites! ». Selon Grice, ce n'est pas sans raisons que les interlocuteurs respectent pareilles maximes. Cela est dû à leur rationalité; il est en effet rationnel de respecter ces maximes pour coopérer et poursuivre effectivement une conversation. Comme je l'ai déjà dit, ce sont les actes illocutoires tentés qui importent dans le discours. Beaucoup d'énonciations ne sont pas littérales ou sérieuses. Quiconque exprime dans un contexte une assertion qui est manifestement fausse, défectueuse ou insincère, étant donné l'arrière-plan conversationnel, n'entend pas alors faire cette assertion mais un autre acte illocutoire avec d'autres conditions de félicité compatibles avec l'arrière-plan. Dans l'approche de Searle et Grice, afin de comprendre les actes illocutoires non littéraux, l'auditeur doit d'abord déterminer l'acte illocutoire littéral et comprendre que le locuteur ne peut alors tout simplement vouloir accomplir cet acte de langage s'il respecte les maximes conversationnelles, étant donné la connaissance factuelle partagée de l'arrière-plan du contexte de l'énonciation. Afin d'expliquer la signification réelle des locuteurs, la pragmatique doit ajouter à la sémantique, conçue 
comme théorie de la signification des énoncés, une théorie des maximes conversationnelles et une analyse des faits pertinents de l'arrière-plan conversationnel. Dans mon approche, les auditeurs infèrent les actes illocutoires non littéraux tentés à partir de la signification dans le contexte de l'énonciation des énoncés utilisés, des conditions de félicité des actes illocutoires exprimés et pertinents et de l'hypothèse du respect des maximes conversationnelles étant donné l'arrière-plan. J'ai généralisé (Vanderveken, 1997) la maxime conversationnelle de qualité de Grice (expliquée comme "Veillez à ce que votre acte illocutoire tenté soit plein de félicité!») ainsi que celle de quantité (expliquée comme «Faites-le aussi fort qu'il le faut pour parvenir à vos fins!»). Comme je l'ai dit plus haut, les conditions de félicité d'un acte illocutoire sont la somme de ses conditions de succès, d'accomplissement sans défaut et de satisfaction. Un acte illocutoire est plus fort qu'un autre quand il possède plus de conditions de félicité. Les deux maximes généralisées s'appliquent dorénavant à tous les genres d'énonciations. La sous-maxime de qualité pour les directives est la suivante: "Veillez à ce que votre directive soit réussie, sans défaut et suivie par l'auditeur! » La sous-maxime de quantité est : "Veillez à ce que votre directive soit aussi forte qu'il le faut! " Les maximes de qualité et de quantité, telles que Grice les a formulées, sont juste des applications de mes maximes dans le cas particulier des assertions de discours à but informatif. Afin de respecter les maximes, les locuteurs doivent bien sélectionner la force et le contenu des actes tentés. Ainsi, ils obéissent aux principes suivants : « Ne donnez pas d'ordre mais faites plutôt une requête à l'auditeur lorsque tout repose sur son bon vouloir! » (sinon votre directive sera défectueuse). " Ne lui demandez pas des choses qu'il ne fera pas!» (sinon votre directive ne sera pas satisfaite). "Ordonnez à l'auditeur quand vous êtes en position d'autorité et que vous voulez vraiment qu'il agisse!» (une demande serait alors trop faible). N'ordonnez ni plus ni moins que ce que vous voulez! » (autrement le contenu de votre directive ne sera pas aussi fort qu'il le faut).

Il existe des formes normales d'inférences menant aux actes illocutoires non littéraux, tentés. Leurs prémisses représentent un nombre fini de faits de l'arrière-plan dont l'existence est présupposée par le locuteur et sur lesquels il entend attirer l'attention des auditeurs. Par conséquent, une approche théorique est en principe compatible avec l'ouverture de l'arrièreplan. Les faits pertinents sont des contenus d'attitudes et reliés aux conditions de félicité. Les auditeurs infèrent les actes illocutoires non littéraux que tentent les locuteurs en exploitant et utilisant les différentes maximes conversationnelles. Comme Grice l'a remarqué, des locuteurs peuvent tranquillement et inostensiblement violer une maxime sans pour autant tenter d'accomplir un acte illocutoire non littéral. Parfois, il y a un conflit entre deux maximes. Le locuteur voudrait bien boire toute la bouteille (maxime de quantité), mais il sait que l'auditeur n'est pas disposé à la lui donner (maxime de qualité). En pareil cas, il vaut mieux simplement faire une demande plus faible, demander un verre plutôt que la bouteille. Il arrive en outre aux locuteurs d'accomplir 
sans le vouloir des actes dépourvus de félicité. Il leur arrive aussi de mentir et de faire, par exemple, des promesses qu'ils ne sont pas capables ou n'ont pas l'intention de tenir afin d'induire en erreur les auditeurs. En pareils cas, les locuteurs ne veulent pas que les auditeurs soient au courant de la violation de la maxime. Pour qu'il y ait exploitation d'une maxime au sens de Grice, il faut que le locuteur veuille que la violation de cette maxime soit manifeste pour les interlocuteurs. Ma notion d'exploitation est une généralisation de celle de Grice. Dans mon optique, un locuteur exploite une maxime conversationnelle quand il entend que l'auditeur reconnaisse des faits de l'arrière-plan qui l'empêcheraient de respecter cette maxime si son acte illocutoire principal était l'acte littéral. Dans le contexte le locuteur est bien coopératif et il peut respecter la maxime en question sans enfreindre une autre. C'est donc qu'il entend alors accomplir principalement un autre acte illocutoire que le littéral et communiquer cette intention. Pareille explication de l'exploitation des maximes permet de caractériser des figures de style. L'ironie et le sarcasme sont des cas particuliers d'exploitation de la maxime de qualité alors que l'euphémisme et l'hyperbole sont des exploitations de la maxime de quantité. Pour comprendre pareilles figures de style, l'auditeur doit reconnaître les faits exploités de l'arrière-plan.

Dans le cas de l'ironie, la signification du locuteur est opposée à la signification de l'énoncé utilisé. L'arrière-plan contient alors des faits exploités incompatibles avec les conditions de félicité de l'acte littéral qui forcent le locuteur à tenter d'accomplir un acte illocutoire non littéral ayant des conditions opposées. L'ironie du locuteur est dirigée vers les composantes de la force et du contenu littéral qui déterminent les conditions manifestement violées de félicité. En disant «Je promets de vous blesser », un locuteur menace ironiquement l'auditeur lorsque l'action envisagée est clairement néfaste pour ce dernier. Son ironie concerne alors une condition préparatoire de l'acte littéral. Une promesse doit être bonne pour l'auditeur. En disant «Je vous remercie pour votre aide ", un locuteur se plaint ironiquement que le locuteur ne lui soit pas venu en aide, lorsqu'il est manifeste qu'il n'a reçu aucune aide et qu'il n'est pas du tout reconnaissant mais au contraire fort insatisfait de cela. L'ironie, dans ce cas, est liée à la fois aux conditions de sincérité et de satisfaction littérales.

Dans le cas des actes de langage indirects, la signification du locuteur est une extension de la signification de l'énoncé utilisé. Le locuteur s'appuie alors sur le fait que l'acte illocutoire littéral n'est manifestement pas assez fort pour atteindre tous ses objectifs linguistiques. La plupart du temps, le locuteur tente manifestement d'atteindre un autre but illocutoire que le littéral sur le contenu propositionnel ou bien il tente d'atteindre le but illocutoire littéral avec un mode spécial non exprimé. Souvent, des conditions préparatoires ou de sincérité non littérales sont manifestement remplies dans l'arrière-plan. Ainsi, on peut promettre indirectement de l'aide en disant «Je vous aiderai». Quand l'indirection est dirigée vers le contenu propositionnel, les conditions de satisfaction de l'acte littéral sont des conditions de félicité de l'acte indirect. Le locuteur affirme parfois que ces conditions sont remplies. Parfois il 
demande à l'auditeur si elles le sont. Dans le premier cas, l'assertion littérale est alors manifestement vraie, dans le second cas, la question littérale a ou pourrait fort bien avoir une réponse positive. Ainsi, nous offrons et même souvent nous promettons indirectement de l'aide en disant "Je pourrais vous aider " (conditions préparatoires de l'acte indirect), "J'ai l'intention de vous aider ", (conditions de sincérité), "Je devrais vous aider ", "Devrais-je vous aider ?" (mode de réalisation du but engageant propre à une promesse). Lors de tels usages idiomatiques, le contenu propositionnel des actes illocutoires indirects fait partie du contenu littéral. Un acte illocutoire indirect est catégorique à chaque fois que ses conditions de félicité non littérales font partie de la connaissance mutuelle d'arrière-plan. Parfois, les intentions du locuteur dépendent de la réponse de l'auditeur. En pareils cas, l'acte de langage indirect est conditionnel. Nous offrons indirectement notre aide à l'auditeur quand nous lui demandons s'il est disposé à recevoir cette aide. De telles offres sont des promesses indirectes qui sont conditionnelles à l'acceptation de l'auditeur.

Mon analyse rend compte des indirections dirigées vers la force aussi bien que vers le contenu. Elle explique les actes illocutoires indirects accomplis grâce à des énonciations non assertives. Contrairement à Sperber et Wilson (1975) dont la notion de pertinence reste vague et informelle, je pense que nous calculons effectivement les actes illocutoires non littéraux tentés. Ils sont minimalement différents des actes de langage littéraux. En général, les actes illocutoires ironiques diffèrent seulement des littéraux par le fait qu'ils ont les composantes opposées aux composantes littérales manifestement incompatibles avec l'arrière-plan. Les actes de langage indirects sont obtenus en ajoutant aux composantes littérales de nouvelles composantes non exprimées déterminant les conditions de félicité manifestement remplies dans l'arrière-plan. Les locuteurs respectent donc les maximes exploitées en faisant de l'ironie et de l'indirection. Les actes illocutoires ironiques ont des conditions de félicité compatibles avec l'arrière-plan (comme l'exige la maxime de quantité). Dans les cas d'indirection, le locuteur entend accomplir, en plus de l'acte littéral, l'acte indirect. Ces deux actes illocutoires sont ensemble aussi forts qu'il le faut pour que le locuteur atteigne tous ses objectifs. Il respecte donc bien alors la maxime de quantité. Les autres figures de style et maximes peuvent être définies selon la même approche. Notons qu'une pragmatique intégrée des actes illocutoires non littéraux est nécessaire pour établir lier les sémantiques synchronique et diachronique en sémiotique. En effet, des modèles récurrents de signification non littérale dans des formes de vie récurrentes d'arrière-plan ont tendance à être lexicalisés après un certain temps. L'on peut ainsi concevoir une théorie du changement de la signification expliquant l'apparition de nouvelles significations littérales (par exemple de métaphores, d'ironies et d'indirections mortes) dans l'histoire de chaque langue. Soit dit en passant, les maximes conversationnelles de qualité et de quantité concernent la nature même des actes illocutoires. Elles ne sont pas relatives à une culture humaine particulière mais sont plutôt des universaux pragmatiques. Selon Grice, elles dérivent 
de la rationalité des locuteurs. Les actes illocutoires sont des moyens naturels d'atteindre des buts linguistiques. Or il existe une certaine raison pratique dans la sélection des moyens appropriés à chaque fin. Des agents rationnels devraient décider d'utiliser en chaque circonstance les meilleurs moyens efficaces disponibles. De même, des locuteurs rationnels devraient tenter d'y accomplir des actes illocutoires à propos. Ainsi, il est raisonnable de respecter la maxime de qualité. Il en va de même pour la maxime de quantité. Tout agent rationnel devrait utiliser des moyens aussi efficaces qu'il le faut dans ses actions verbales aussi bien que non verbales. Or à chaque but linguistique correspond un acte illocutoire unique servant à atteindre ce but pleinement et de la façon la plus effective. Un locuteur qui tenterait d'accomplir un acte illocutoire plus faible ou plus fort n'agirait pas avec la plus grande efficacité pour parvenir à son but.

Searle (1982) a souligné d'autres difficultés importantes pour la théorie du discours: les interlocuteurs qui dialoguent n'ont souvent aucun objectif conversationnel; ils changent librement le genre et le sujet du dialogue; leurs énonciations ratées, sans félicité et totalement impertinentes n'empêchent parfois pas leur dialogue de continuer. De plus, l'arrière-plan conversationnel est indéfiniment ouvert et par conséquent impossible à décrire exhaustivement. Enfin, leur intentionnalité discursive est irréductiblement collective. J'ai répliqué à Searle (Vanderveken, 1997, 2001), en faisant les considérations suivantes. Bien sûr, les locuteurs sont engagés dans des formes de vie sociales, et leurs objectifs sont fort souvent extralinguistiques. Ils communiquent alors afin de coordonner intelligemment leurs actions non verbales. Je suis d'accord avec Wittgenstein (1958) et Searle (1982), qu'il n'existe pas de véritable théorie possible de tous les jeux de langage possibles. Il y a d'innombrables genres de formes de vie et d'objectifs que nous pouvons partager en tant qu'être humain. Conséquemment, il existe "d'innombrables genres » de jeux de langage que nous pouvons pratiquer. Comme Wittgenstein le dit, «[...] cette multiplicité n'est pas quelque chose de fixe, de donnée une fois pour toutes; mais de nouveaux types de langages, de nouveaux jeux de langage, nous pouvons le dire, en viennent à exister, alors que d'autres deviennent obsolètes et tombent dans l'oubli» (Wittgenstein, $1958: 23$ ).

Pourtant, en tout dialogue, les locuteurs entendent accomplir des actes illocutoires pertinents afin de contribuer à des échanges verbaux comme les présentations, les justifications, les débats, les compromis et les ententes qui sont des actes de langage de niveau supérieur à but conversationnel. La logique du discours peut analyser la structure et la dynamique de tels jeux de langage dont la conduite est régie par des systèmes de règles constitutives. Ma taxonomie postule quatre buts conversationnels correspondant aux quatre directions possibles d'ajustement entre les mots et les choses. Les discours avec la direction d'ajustement des mots aux choses (comme les rapports, les entrevues, les nouvelles et les interrogations) ont un but descriptif: ils servent à décrire comment les choses sont dans le monde. Les discours avec la direction d'ajustement 
des choses aux mots (comme les négociations, les marchandages, les paris, les tentatives d'arrangement et les sermons) ont un but délibératif: délibérer comment agir dans le monde. Les discours avec la double direction d'ajustement (les inaugurations, les permis, les baptêmes, les investitures et les classifications) ont un but déclaratoire: ils servent à faire des choses par déclaration. Finalement, les discours sans direction d'ajustement (les salutations, les accueils, les éloges et les protestations) ont un seul but expressif: ils servent seulement à exprimer les attitudes communes des interlocuteurs. Les locuteurs compétents sont tous capables d'atteindre les buts conversationnels à cause de leur intentionnalité. Il y a une correspondance biunivoque entre les buts conversationnels et les directions possibles d'ajustement entre les mots et les choses, car les véritables protagonistes de chaque dialogue sont les interlocuteurs qui y contribuent successivement. Dans un contexte isolé d'énonciation, le locuteur est actif alors que l'auditeur est passif. Aucun locuteur ne peut engager quelqu'un d'autre à agir par son énonciation personnelle. Il peut seulement s'engager lui-même ou donner une directive à un autre agent. C'est pourquoi le langage distingue naturellement deux buts illocutoires avec la direction d'ajustement des choses aux mots. Quand l'énonciation est engageante, la responsabilité de changer le monde repose sur le locuteur; quand elle est directive, elle repose sur l'auditeur. Cependant, la situation est entièrement différente lors de la poursuite d'une conversation. Tout auditeur est un locuteur potentiel qui peut parler à son tour et faire sa propre contribution. Ainsi, lors d'une délibération, les auditeurs peuvent répliquer aux locuteurs qui leur donnent des directives et font des offres. Ils peuvent par la suite accepter, refuser ou faire des contre-offres. Souvent, les engagements des locuteurs sont conditionnels aux engagements futurs des interlocuteurs. Par conséquent, il y a un seul but conversationnel alors qu'il y a deux buts illocutoires différents avec la direction d'ajustement des choses aux mots. Les délibérations servent autant à engager les interlocuteurs qu'à leur donner des directives; ils servent à déterminer des actions réciproques à faire dans le monde.

Toutes les forces ayant le même but illocutoire ne jouent pas le même rôle dans l'usage du langage. Il vaut parfois mieux conseiller qu'ordonner. De même, tous les types de dialogue ayant le même but conversationnel n'ont pas la même fonction. Parfois, il vaut mieux expliquer et justifier sa position plutôt que de la présenter unilatéralement. Ma typologie décompose les types discursifs en d'autres composantes que leurs buts conversationnels. Beaucoup ont un mode caractéristique d'atteinte du but conversationnel, qui requiert l'usage de certains moyens ou une façon particulière de procéder. La plupart des modes imposent une certaine séquence d'actes illocutoires majeurs. Dans les contrats écrits, les parties doivent s'engager à des actions réciproques futures et s'entendre officiellement en donnant leur signature. De nombreux types de discours imposent des conditions à leur thème. Une entrevue d'embauche doit décrire les qualités professionnelles de l'interviewé. Les conditions thématiques déterminent à la fois la force et le contenu propositionnel des actes illocutoires 
majeurs. Les délibérations d'un jury doivent aboutir à un verdict. De nombreux types de discours exigent que des conditions préparatoires soient remplies dans l'arrière-plan. Par exemple, lors d'une consultation médicale, on présuppose que la personne consultée a des aptitudes en médecine et que celui qui consulte veut des conseils concernant sa santé. Un discours est défectueux quand ses conditions préparatoires sont violées. Finalement, beaucoup de types discursifs exigent que les protagonistes expriment des attitudes communes. Lors d'échanges de salutations, les interlocuteurs reconnaissent courtoisement la présence des autres à leur approche. Dans ma typologie deux jeux de langage ont le même type discursif quand ils ont les mêmes buts conversationnels, les mêmes modes d'atteinte de ces buts, les mêmes conditions thématiques et les mêmes conditions d'arrière-plan et de sincérité.

J'ai formulé une définition récursive de l'ensemble de tous les types possibles de discours à but conversationnel et j'ai systématiquement analysé leurs conditions de félicité. Les quatre types primitifs de discours sont les types les plus simples correspondant aux quatre buts conversationnels : la description, la délibération, la déclaration et l'expression; ils sont communs à tous les jeux de langage respectivement descriptifs, délibératifs, déclaratoires, et expressifs. Les autres types de discours plus complexes sont obtenus en ajoutant de nouvelles composantes aux types primitifs. Ainsi le type de la négociation possède le mode particulier d'atteinte du but délibératif qu'est la concertation : les protagonistes doivent se concerter lors de leur délibération. Le type du marchandage a la condition thématique additionnelle que les protagonistes négocient l'achat et la vente de certaines choses. Le type des pourparlers de paix a la condition d'arrière-plan particulière que les négociateurs représentent les partis belligérants et qu'ils sont autorisés à conclure la paix. Finalement, les tentatives de règlement à l'amiable ont la condition de sincérité particulière que les négociateurs expriment alors leur volonté d'arriver à un arrangement sans animosité.

Chaque composante de type discursif détermine une condition de succès particulière correspondant à une règle constitutive à suivre pour tenir ce type de discours. Les protagonistes réussissent à poursuivre un discours d'un type particulier durant un intervalle de temps quand, premièrement, leur thème satisfait aux conditions thématiques de leur type, deuxièmement, ils atteignent le but conversationnel sur le thème avec le mode d'atteinte requis, troisièmement, ils présupposent que les conditions d'arrière-plan requises sont remplies et, finalement, ils expriment les attitudes communes requises par les conditions de sincérité. Ainsi, les locuteurs marchandent quand ils délibèrent sur un achat et une vente (but discursif et conditions thématiques), ils négocient en effectuant des offres, des contre-offres, des accords et des refus de commerce (mode d'atteinte du but délibératif), ils tiennent pour acquis qu'ils sont acheteurs et vendeurs potentiels (conditions d'arrière-plan), et ils expriment leur volonté commune de faire des affaires (conditions de sincérité). Quand un type de discours possède plus de composantes qu'un autre, il a des conditions de succès plus fortes. On ne peut poursuivre ce type de discours sans poursuivre un discours de l'autre 
type. Ainsi les séances de marchandage, les pourparlers de paix et les tentatives de règlement à l'amiable sont des négociations, et les négociations des délibérations.

Remarquez que les conditions de succès des discours ne sont pas la somme des conditions de succès de leurs actes illocutoires successifs. Comme Roulet (1990) l'a bien souligné, un dialogue ne peut pas être décomposé directement en la suite des actes illocutoires individuels instantanés tentés lors de ses énonciations successives. Avant tout, un dialogue est une suite d'actes de discours de deuxième niveau comme des salutations, présentations, prises de position, explications, illustrations, répliques, répétitions, commentaires, résumés, prises de décision et conclusions qui durent un certain intervalle de temps. Pareilles interventions discursives sont des unités de discours d'articulation supérieure plus complexes que les simples actes illocutoires auxiliaires correspondant aux énonciations successives d'énoncés. En cela, Wittgenstein avait bien raison de dire que la poursuite d'un discours ressemble à la pratique d'un jeu. Prenons l'exemple d'une partie d'échecs. Rappelons que Saussure (1916) a comparé la langue au jeu d'échecs. Une partie d'échecs est plus que la suite des coups successifs des deux adversaires. Elle consiste plutôt en une suite d'échanges de plusieurs coups comme le développement initial du jeu de chaque joueur, leurs mouvements de pièces pour se concentrer et disposer d'une force supérieure sur l'échiquier, les attaques et les contre-attaques pour gagner l'avantage et les tentatives d'échec et mate. De même un discours complet tel qu'un débat théorique consiste en différents échanges linguistiques comme la présentation des participants, l'introduction à l'état de la question, les thèses de chacun, leurs explications, justifications, clarifications et illustrations, les objections, leurs critiques et tentatives de réfutation d'autres thèses, les résumés et les conclusions. D'un point de vue théorique, il faut distinguer en tout discours pareilles interventions des actes illocutoires auxiliaires plus simples tentés lors de chaque énonciation d'énoncés. Comme Searle, je pense que ces interventions sont des actes illocutoires de deuxième niveau dont la conduite exige une suite de plusieurs actes illocutoires instantanés. Les locuteurs font seuls ou avec d'autres plusieurs énonciations en chaque intervention.

Comme les linguistes de Genève, je distingue donc en tout discours des unités de niveaux différents. Les interventions y ont une fonction propre. À une certaine étape conversationnelle, les locuteurs comprennent qu'il leur faut développer leurs positions, qu'ils ont besoin d'argumenter, d'expliquer et de clarifier leurs idées, de se justifier ou de répliquer à des énonciations précédentes. Ainsi de nombreux types de discours imposent un ordre à la suite de leurs interventions, de même que de nombreuses interventions imposent un ordre à la suite de leurs actes illocutoires instantanés. Dans une théorie scientifique, il y a des interventions à but descriptif (la formulation des hypothèses, les justifications), à but déclaratoire (les axiomatisations et démonstrations) et à but délibératif (les expériences et tentatives de confirmations empiriques). Dans une démonstration, les locuteurs posent les prémisses avant de conclure. 
Dans mon optique, la poursuite d'un type de discours est bien un processus dont le développement est pourvu d'une certaine structure. Les interlocuteurs accomplissent chacun à leur tour des actes illocutoires individuels instantanés afin de contribuer à des interventions qui jouent un rôle dans la conversation en cours.

Ainsi l'intentionnalité interlocutoire à l'œuvre dans la poursuite d'un discours est-elle supérieure à la somme des intentionnalités à l'œuvre dans les actes illocutoires individuels qui en font partie. La poursuite des discours à but conversationnel requiert seulement la réussite et parfois aussi la félicité des actes illocutoires capitaux (Foster, 1984) de premier et de second niveau qui sont centraux dans ces discours. La forme et le moment d'accomplissement des actes illocutoires capitaux dépendent du type conversationnel et du thème du discours. Tout d'abord, chaque discours doit contenir la suite d'interventions capitales que requiert le mode de réalisation du but conversationnel propre à son type. Beaucoup de types discursifs exigent une présentation initiale de leurs protagonistes. Lors d'une entrevue d'embauche il faut que des questions soient posées à l'interviewé et qu'il tente d'y répondre. Pareilles interventions constitutives sont elles-mêmes pourvues d'un type et d'un thème; ce sont des sous-discours qui peuvent être tenus séparément. Il arrive que la suite des questions posées lors d'une entrevue soit publiée séparément dans un journal. Ensuite, chaque intervention capitale doit contenir la suite des actes illocutoires individuels qui sont centraux en cette intervention. Pour réussir une intervention il faut y accomplir des actes illocutoires individuels capitaux ayant la direction d'ajustement propre au but conversationnel. Il y a des assertions capitales en toute description comme il y a des actes engageants et directifs capitaux en toute délibération. En outre les forces et les contenus des actes illocutoires individuels capitaux doivent satisfaire les conditions thématiques du type discursif de l'intervention relativement à son thème. Lors d'un marchandage, les protagonistes peuvent faire de nombreuses remarques superflues qui ne sont pas pertinentes. Mais ils doivent à tout le moins faire des offres d'achat ou de vente et essayer de parvenir à un accord commercial. Parfois, un acte illocutoire capital met fin au discours. Un accord de vente est une façon plutôt agréable de conclure un marchandage. Mais un désaccord peut aussi y mettre fin. Les délibérations d'un jury lors d'un procès doivent se terminer par un verdict. Les actes illocutoires d'un discours n'ont pas tous la même importance. Seuls les actes illocutoires capitaux importent vraiment. Leur accomplissement est essentiel. Les actes illocutoires superflus peuvent être ratés et sans pertinence. Ils n'empêchent pas les discours à but conversationnel de continuer. Les interlocuteurs qui entendent tenir pareils discours doivent cependant garder leur intention conversationnelle commune durant leur poursuite. La maxime de pertinence impose donc à présent de fortes contraintes à la structure et au développement de ces discours. Leurs protagonistes doivent en effet coopérer et suivre les règles constitutives en effectuant au bon moment des contributions capitales appropriées. Ils ne peuvent alors changer d'objectifs linguistiques sans arrêter ou faire échouer la conversation en cours. 
Les discours avec une direction non vide d'ajustement ont des conditions de satisfaction. Comme on peut s'y attendre, leur satisfaction dépend de la satisfaction de leurs actes illocutoires capitaux. Les descriptions sont exactes quand leurs assertions capitales sont vraies. Les délibérations sont respectées quand leurs interlocuteurs tiennent leurs engagements capitaux et suivent leurs directives capitales. Enfin les discours à but déclaratoire sont satisfaits quand leurs interlocuteurs réussissent leurs déclarations capitales. Lors d'un discours les interlocuteurs raisonnent et ils peuvent changer d'attitudes et réviser leurs actes illocutoires antérieurs. Ce qui compte est leur position finale. Des interlocuteurs différents peuvent ne pas s'entendre et même se contredire. Mais souvent ils argumentent et essaient de se convaincre. Il arrive que des interlocuteurs changent de position en admettant des critiques. Parfois ils procèdent à un vote pour prendre une décision commune. Quand les protagonistes parviennent à un accord final, leur dialogue s'achève de façon heureuse. Ils s'entendent alors sur ce qui se passe dans le monde ou sur ce qu'il faut y faire. Cependant, la réussite d'un discours ne requiert pas l'accord final. Bien des marchandages se terminent sans achat. En cas de désaccord, le discours contient des actes illocutoires capitaux simultanément insatisfaisables. La théorie du succès requiert en général moins que la félicité et la bonne performance. Bien des actes illocutoires réussis lors d'une énonciation sont mal faits, faits à des moments inappropriés, défectueux, insincères ou insatisfaits. Il en va de même pour les discours entiers. Il arrive qu'une consultation d'expert soit mal faite. Les participants n'ont pas parlé des choses importantes et ils se sont basés sur des données erronées. Il se peut même que l'expert soit un imposteur. Beaucoup de discours réussis et bien faits contiennent des actes illocutoires capitaux insatisfaits. De bonnes consultations d'experts peuvent aboutir à de mauvaises expertises. Il arrive qu'une bonne annonce de publicité ne fasse pas augmenter les ventes.

Jusqu'à présent, les théoriciens de la conversation ont négligé les buts conversationnels. Beaucoup ont distingué les bons et les mauvais discours de certains genres. Mais peu ont reconnu que les dialogues sont des actes illocutoires conjoints d'ordre supérieur pourvus de conditions de félicité. Ceux qui ont analysé les conversations ordinaires (Schegloff) ont empiriquement analysé des modèles récurrents d'interaction verbale comme les règles gouvernant le tour de parole entre interlocuteurs. Certains linguistes (van Eemeren, Jucker, Atkinson et Drew) ont analysé des dialogues à but conversationnel d'un type particulier comme les argumentations, les procès en cour de justice, les entrevues d'embauche, les journaux télévisés et les leçons à l'école. Les philosophes des sciences ont étudié la nature du discours scientifique et des philosophes de la religion celle du discours religieux. Les logiciens ont étudié la nature des démonstrations mathématiques (la théorie de la preuve). Pareilles investigations sont restreintes mais fort utiles. D'autres ont adopté une approche plus générale; ils ont analysé divers types de discours. Les linguistes de Genève (Eddy Roulet) utilisent le modèle hiérarchique des échanges. Hans Kamp a 
construit une théorie formelle de la représentation des discours permettant de caractériser les thèmes et résoudre dynamiquement les anaphores et les présuppositions. Sperber et Wilson ont étudié la pertinence. Mais la plupart ont ignoré les types discursifs. J'entends renforcer l'approche théorique sur la conversation en intégrant les résultats obtenus en syntaxe, sémantique et pragmatique formelles dans une pragmatique plus générale du discours utilisant des formalismes logiques pour caractériser la structure et la dynamique des jeux de langage à but conversationnel. Voir à ce sujet mon prochain livre, Speech Acts in Dialogue. Une pragmatique du discours trop dépendante de la terminologie du langage ordinaire et dépourvue de vocabulaire technique et de formalisme est à éviter.

Quelles ressources le langage met-il à notre disposition afin de dialoguer ? Il existe de nombreux verbes conversationnels, notamment des performatifs (les expositifs de Austin) ainsi que des connecteurs discursifs comme "par conséquent ». K. MacQueen et moi avons procédé à une analyse lexicale raisonnée des verbes conversationnels de langue anglaise nommant des types discursifs sur la base de ma typologie. Il n'y a pas de correspondance biunivoque entre les types discursifs et les verbes conversationnels, tout comme il n'y a pas de correspondance biunivoque entre les forces illocutoires et les verbes performatifs. Des verbes comme «marier» et «parier» nomment à la fois un type de discours et un acte illocutoire individuel qui y joue un rôle capital. Des verbes conversationnels comme « répondre » et « conclure » nomment des interventions discursives sans but conversationnel propre. Une réponse et une conclusion peuvent avoir n'importe quel but. Certains verbes conversationnels comme « débattre » et "argumenter» sont ambigus. Les argumentations en faveur ou à l'encontre d'une thèse sont descriptives, celles en faveur ou à l'encontre d'une ligne de conduite sont délibératives. Lorsqu'un verbe conversationnel est ambigu, les types de discours qui y correspondent partagent certains traits. Ceux qui argumentent tentent de se convaincre. La volonté commune de persuader les autres protagonistes est un mode particulier de réalisation du but conversationnel propre à toute argumentation.

Afin de contribuer aux fondements de la pragmatique formelle du discours, il convient d'analyser la nature propre aux attitudes et actions conjointes en philosophie de l'esprit et de l'action, lesquelles ne sont pas réductibles, comme Searle l'a montré, à des sommes d'attitudes et d'actions individuelles. Les dialogues à but conversationnel sont des actes illocutoires conjoints de niveau supérieur dont la poursuite requiert de l'intentionnalité constitutive conjointe et de la coopération entre les interlocuteurs. Comment les protagonistes en viennent-ils à partager leur intention conversationnelle et à coordonner leurs contributions? Il leur arrive de négocier le type conversationnel. Selon quels principes choisissent-ils leurs actes illocutoires et la façon de les accomplir ? Comment l'arrière-plan conversationnel et les énonciations antérieures interviennent-ils? Vu notre rationalité, nous révisons et révoquons des attitudes et actes illocutoires antérieurs lorsqu'ils s'avèrent défectueux ou impossibles 
à satisfaire. Les logiques paraconsistente et non monotone (da Costa, Prakke $\&$ Vreeswijk) sont utiles pour indiquer comment nous raisonnons quand des informations antérieures s'avèrent incomplètes, fausses ou même incohérentes. Pouvons-nous les utiliser ? Il convient de réfléchir à cette question.

J'entends en outre exploiter des développements récents en logique dynamique et dialogique (Lorenz, Hintikka, van Benthem, Rahman) afin de caractériser la dynamique propre aux dialogues à but conversationnel. Les dialogues de la logique dialogique traditionnelle visent à vérifier si une hypothèse initiale est ou non valide. Le premier locuteur est censé défendre son hypothèse, le second la contester. Il convient d'utiliser la logique illocutoire afin de caractériser avec exactitude les différents coups permis aux interlocuteurs en de tels dialogues. Toutes les défenses ne sont pas des assertions catégoriques. Certaines sont des assertions conditionnelles. En outre, les attaques sont des directives. Avec M. Paquette j'utiliserai la théorie des jeux (von Neumann) afin d'élaborer une théorie qualitative de la décision définissant une valeur de choix d'une façon comparable à la théorie bayésienne en termes d'ensemble hiérarchisé de préférences et de structure de probabilité (Doyle) plutôt que de fonctions de probabilité. Il s'agit de définir le succès (l'existence d'une solution) comme un équilibre de Nash pour les dialogues simples à but conversationnel. J'envisage aussi d'utiliser la théorie de la représentation du discours afin de mieux analyser le thème conversationnel ainsi que la dépendance vis-à-vis de l'arrière-plan des actes illocutoires tentés lors de chaque contribution. En considérant les énonciations dans le cadre des échanges, la pragmatique du discours offre une nouvelle perspective à la théorie de la signification qui devient plus dynamique et collective. Non seulement la théorie peut dorénavant mieux déterminer la force et le contenu des énonciations de tout genre en tenant compte des énonciations antérieures et des faits de l'arrière-plan. Mais les interlocuteurs peuvent aussi clarifier la signification d'énonciations antérieures et même leur donner une nouvelle interprétation. Enfin la théorie peut déterminer les actes illocutoires conjoints auxquels les interlocuteurs entendent contribuer en faisant leurs énonciations individuelles instantanées.

\section{Bibliographie}

Austin, J. L. How to Do Things with Words, Clarendon Press, 1962.

Belnap, N. et al. Facing the Future Agents and Choices in our Indeterminist World, Oxford University Press, 2001.

Belnap, N. \& Steel T. The Logic of Questions and Answers, Yale University Press, 1976. Cherniak, C. Minimal Rationality, MIT Press, Bradford Books, 1986.

Cocchiarella, N. «Formally Oriented Work in the Philosophy of Language", in J. Canfield, (dir.) Philosophy of Meaning, Knowledge and Value in the $20^{\text {th }}$ Century, Routledge, 1997, p. 39-45.

Van Eemeren, F. H. et R. Grootendorst. A Systematic Theory of Argumentation, Cambridge University Press, 2004.

Fotion, N. «Master Speech Acts ", Philosophical Quaterly, 21, 1984, 232-243.

Frege, G. Logical Investigations, Yale University Press, 1977. 


\section{Philosophiques / Automne 2007}

Goldman, A. I. A Theory of Human Action, Princeton University Press, 1970.

Grice, H. P. Logic and Conversation, in P. Cole \& J. Morgan (ed.) Syntax and Semantics, vol. 3, New York Academic Press, 1976, p. 41-58.

Harrah, D. «The Logic of Questions » in D. Gabbay \& F. Guenthner (eds.) Handbook of Philosophical Logic, vol. 2, Reidel, 1984.

Kamp, H. \& U. Reyle. From Discourse to Logic, Kluwer, 1993.

Kaplan, D. Demonstratives. in J. Almog et al, Themes from Kaplan, Oxford University Press, 1989, p. 481-563.

Montague, R. Formal Philosophy, Yale University Press, 1974.

Prior, A. N. Past, Present, Future, Oxford, Clarendon Press, 1967.

Resher, N. The Logic of Commands Routledge \& Kegan Paul, 1966.

Roulet, E. 1992. "On the Structure of Conversation in Negotiation », in J. R. Searle et al. On Searle and Conversation, Benjamins, 1992, p. 91-99.

Saussure (de), F. Cours de linguistique générale, Payot, 1995.

Searle, J. R. 1992. "Conversation », in J. R. Searle et al. On Searle and Conversation, Benjamins, 1992, p. 7-29.

Searle, J. R. \& D. Vanderveken. Foundations of Illocutionary Logic, Cambridge University Press, 1985.

Sperber, D. \& D. Wilson. Relevance, Harvard University Press, 1986.

Thomason, R. Combinations of Tense and Modality, in D. Gabbay \& F. Guenthner (ed.) Handbook of Philosophical Logic, vol. 2, 1984, p. 135-165.

Vanderveken, D. Meaning and Speech Acts, vol. 1-2, Cambridge University Press, 19901991.

—. 1997. "La logique illocutoire et l'analyse du discours » in D. Luzzati et al. (dir.) Le dialogique, Peter Lang, p. 59-94

- 1997. "Formal Pragmatics of Non Literal Meaning ", Linguistische Berichte, 8, p. 324-341.

—. 2001. "Illocutionary Logic and Discourse Typology ", Revue internationale de philosophie, $\mathrm{n}^{\circ} 216$, p. 243-255.

- 2004. "Success, Satisfaction and Truth in the Logic of Speech Acts and Formal Semantics ", in Davis, S. \& B. Gillan (eds.), Semantics A Reader, Oxford University Press, p. 710-734.

- 2005. "Attempt, Success and Action Generation: A Logical Study of Action Generation ", in D. Vanderveken (dir.), Logic, Thought \& Action, Springer, p. 315-342.

- 2006. "Croyances, certitudes et rationalité minimale des agents humains ", Psychologie de l'interaction, $\mathrm{n}^{\circ} 21$ et 22, p. 13-44.

_- 2006. "Fondements de la logique des attitudes" dans le numéro spécial Language and Thought de Manuscrito, vol. 29, $\mathrm{n}^{\circ}$ 3, p. 351-98.

Wittgenstein, L. Philosophical Investigations, Blackwell, 1958.

Von Neumann \& Morgenstern. Theory of Games and Economic Behavior, Princeton, University Press, 1944. 